\title{
Adaptive Asymptotic Tracking Control for a Class of Uncertain Switched Systems via Dynamic Surface Technique
}

\author{
Xuemiao Chen, ${ }^{1,2}$ Qianjin Zhao, ${ }^{3}$ Chunsheng Zhang, ${ }^{4}$ and Jian Wu $\mathbb{i D}^{4}$ \\ ${ }^{1}$ College of Mathematics and Big Data, Anhui University of Science \& Technology, Huainan, China \\ ${ }^{2}$ State Key Laboratory of Mining Response and Disaster Prevention and Control in Deep Coal Mines, \\ Anhui University of Science \& Technology, Huainan, China \\ ${ }^{3}$ College of Computer Science and Engineering, Anhui University of Science \& Technology, Huainan, China \\ ${ }^{4}$ The University Key Laboratory of Intelligent Perception and Computing of Anhui Province, Anqing Normal \\ University, Anqing, China
}

Correspondence should be addressed to Jian Wu; jwu2011@126.com

Received 5 June 2019; Revised 7 September 2019; Accepted 14 September 2019; Published 27 October 2019

Academic Editor: Danilo Comminiello

Copyright ( $\odot 2019$ Xuemiao Chen et al. This is an open access article distributed under the Creative Commons Attribution License, which permits unrestricted use, distribution, and reproduction in any medium, provided the original work is properly cited.

A novel adaptive tracking control scheme is proposed for a class of uncertain nonlinear switched systems with perturbations in this paper. The common Lyapunov function method is introduced to handle the switched system in the design process of the desired adaptive controller. In addition, a dynamic surface control method is proposed by employing a nonlinear filter such that the "explosion of complexity" problem existing in the conventional backstepping design can be overcome. Under the presented adaptive controller, all the closed-loop signals are semiglobally bounded, and especially the output signal of the controlled system can follow the given reference signal asymptotically. To show the availability of the presented control scheme, a simulation is given in this paper.

\section{Introduction}

Switched systems, as a typical class of hybrid systems, have attracted many researchers' sights in the past decades (e.g., see [1-14]) since many physical systems can be mathematically modeled by the switched systems. Owing to the large-scale applications, the research on switched systems never stops and great achievements have been made (e.g., see [1-6]). The stabilization problem for a class of slowly switched systems based on unstable subsystems is studied in [6]. The optimal control scheme of the switching positive system is presented in [7]. The stabilization problem of switched linear systems with mode-dependent average dwell time is addressed in [8]. Most significant issues on switched systems have been acquired for multitudinous switched systems under arbitrary switching or constraint switching (e.g., see [8-11]), and references therein.

Adaptive control of uncertain nonlinear systems has achieved significant research results (e.g., see [15-25]) including adaptive feedback linearization [15], adaptive backstepping [16], immersion and invariance adaptive control [17], and adaptive neural network/fuzzy-logic control $[21,25]$. Very recently, some novel adaptive control schemes have been established for uncertain systems with the unknown control directions or the parameter estimator triggering [26-28]. However, the "explosion of complexity" problem exists in the repeated differentiations of virtual control variables $[18,19]$ in the backstepping approach. Thus, with the system order increasing, the computation of the backstepping controller will be more complicate.

To overcome the "explosion of complexity" problem in the backstepping design process, for uncertain strict-feedback/nonstrict-feedback nonlinear systems, the dynamic surface control (DSC) technique is established (e.g., see [29-38]). In literature studies [29, 30], a virtual control law is designed in each design step by using a low-pass filter, thus it can avoid the derivative of the virtual controllers. Another advantage of the DSC approach is that it can reduce the 
requirement on the smoothness of plant functions and desired signal obviously. Therefore, the DSC technique has a large-scale application in the process of designing simplified adaptive controllers for uncertain nonlinear systems. The adaptive robust DSC with composite adaptation laws is designed for the uncertain system with the semistrict feedback form in [31]. Based on multiple models, the result is applied to enhance the transient response of an adaptive DSC system in [32]. An adaptive DSC scheme is presented by using composite learning to ensure parameter convergence without the persistent excitation condition in [33]. Various adaptive backstepping schemes using neural networks (NNs) or fuzzy logic systems as approximators are proposed to deal with the time delays, dynamic uncertainties, and output dead zone for stochastic large-scale nonlinear systems in [34-37]. Very recently, a novel DSC method with the nonlinear filter is proposed for a class of uncertain systems, and the asymptotic tracking control performance is achieved in [38].

In the above discussions, some adaptive DSC schemes have been developed for uncertain switched systems. Nevertheless, these control schemes can just guarantee the semiglobal boundedness for the controlled systems, and the asymptotic stability cannot be achieved since the stability analysis cannot be completed by the conventional DSC technique with a linear low-pass filter. In this paper, we try to address this issue by employing the DSC method with a nonlinear filter. The main contributions of this work are summarized as following.

(i) As far as we know now, this is the first work to address the asymptotic tracking control problem for uncertain switched systems by using the DSC method to solve the "explosion of complexity" problem existing in the conventional backstepping design.

(ii) By introducing the DSC method with a nonlinear filter proposed in [38], the desired controller is developed based on the common Lyapunov function, and then the stability analysis of the closed-loop switching control system is completed according to Barbalat's lemma.

This paper is organized as follows. The problem statement and some preliminaries are introduced and adaptive DSC scheme with a detailed stability analysis are presented in Section 2. Then, a simulation example is given in Section 3. A conclusion is drawn in Section 4.

\section{Problem Statement and Main Result}

2.1. Problem Statement and Some Preliminaries. Taking the following class of uncertain strict-feedback nonlinear switched systems into account,

$$
\left\{\begin{array}{l}
\dot{x}_{i}=x_{i+1}+\theta_{i} f_{i, \varrho(t)} \overline{\left(x_{i}\right)}+d_{i}(t), \quad i=1, \ldots, n-1, \\
\dot{x}_{n}=u+\theta_{n} f_{n, \varrho(t)} \overline{\left(x_{n}\right)}+d_{n}(t), \\
y=x_{1},
\end{array}\right.
$$

where $\bar{x}_{i}=\left[x_{1}, \ldots, x_{i}\right]^{T} \in R^{i}, i=1, \ldots, n$ are the states of the system, $u \in R$ is the control input, and $y \in R$ is the system output. For $i=1, \ldots, n, \theta_{i}$ are the unknown constants, $d_{i}(t)$ are the perturbations, and $f_{i}(\cdot): R^{n} \longrightarrow R$ are the known continuously differentiable functions. $\varrho(t):[0, \infty)$ is the switching signal, and $p$ denotes the number of subsystems in the switched system.

The control objective of this paper for system (1) is to design an adaptive DSC law $u$ such that the output $y(t)$ asymptotically tracks a desired trajectory $y_{r}(t)$, and the boundedness of all the signals in the closed-loop system is guaranteed.

Assumption 1. The desired trajectory $y_{r}(t)$ and its derivatives $\dot{y}_{r}(t)$ and $\ddot{y}_{r}(t)$ are bounded and available.

Assumption 2. The perturbations $d_{i}(t), i=1, \ldots, n$ are bounded, i.e., $\left|d_{i}(t)\right| \leq W_{i}$ with the constants $W_{i}>0$.

Lemma 1 (see [39]). For any $\varepsilon>0$ and $z \in R$, the following inequality can be obtained:

$$
0 \leq|z|-\frac{z^{2}}{\sqrt{z^{2}+\varepsilon^{2}}}<\varepsilon
$$

2.2. Adaptive Controller Design. The presented adaptive DSC scheme is similar to the backstepping technique, and it contains $n$ steps as follows. Define the estimate error $\widetilde{*}=*-\hat{*}$, where $\hat{*}$ is the estimate of $\hat{*}$. Let $\varepsilon_{i}=\left|\theta_{i}\right|, i=1, \ldots, n$.

Step 1. The first surface error is defined as $z_{1}=x_{1}-y_{r}$, and the time derivative of $z_{1}$ is

$$
\dot{z}_{1}=\dot{x}_{1}-\dot{y}_{r}=x_{2}+\theta_{1} f_{1, \varrho(t)} \overline{\left(x_{1}\right)}+d_{1}(t)-\dot{y} r .
$$

Then, the Lyapunov function candidate $V_{1}$ is defined as

$$
V_{1}=\frac{1}{2} z_{1}^{2}+\frac{1}{2 \gamma_{1}} \widetilde{\varepsilon}_{1}^{2}+\frac{1}{2 \beta_{1}} \widetilde{W}_{1}^{2},
$$

where $\gamma_{1}$ and $\beta_{1}$ are positive design parameters.

Considering equations (1)-(4), the following equation can be obtained:

$$
\begin{aligned}
\dot{V}_{1}= & z_{1}\left(x_{2}+\theta_{1} f_{1, \varrho(t)} \overline{\left(x_{1}\right)}+d_{1}(t)-\dot{y}_{r}\right)-\frac{1}{\gamma_{1}} \widetilde{\varepsilon}_{1} \dot{\hat{\varepsilon}}-\frac{1}{\beta_{1}} \widetilde{W}_{1} \dot{\hat{W}}{ }_{1} \\
= & z_{1}\left(x_{2}+\theta_{1} f_{1, \varrho(t)} \overline{\left(x_{1}\right)}+d_{1}(t)-\dot{y}_{r}+\alpha_{1}-\alpha_{1}\right)-\frac{1}{\gamma_{1}} \widetilde{\varepsilon}_{1} \dot{\hat{\varepsilon}} \\
& -\frac{1}{\beta_{1}} \widetilde{W}_{1} \dot{\hat{W}}_{1} .
\end{aligned}
$$

On the basis of $f_{i, \varrho(t)}$, we can obtain the following inequality: 


$$
\left|f_{i, \varrho(t)}\right| \leq \sqrt{\sum_{j=1}^{p} f_{i, j}^{2}}
$$

The above inequality is introduced to design the desired controller by employing the common Lyapunov function method. In order to develop an adaptive controller without the switching signal $\varrho(t)$, the subsystem function $f_{i, \varrho(t)}$ is bounded by an upper bound function which is without the switching signals.

Let $D_{i}=\sqrt{\sum_{j=1}^{p} f_{i, j}^{2}}$. The following inequality can be obtained:

$$
\begin{aligned}
z_{1} \theta_{1} f_{1, \varrho(t)} & \leq\left|z_{1}\right|\left|\theta_{1}\right|\left|f_{i, \varrho(t)}\right| \\
& \leq\left|z_{1}\right|\left|\theta_{1}\right| D_{1} \\
& \leq \varepsilon_{1} \frac{z_{1}^{2} D_{1}^{2}}{\sqrt{z_{1}^{2} D_{1}^{2}+\sigma(t)^{2}}}+\varepsilon_{1} \sigma(t),
\end{aligned}
$$

and the following inequality can be obtained by using Assumption 2

$$
\begin{aligned}
z_{1} d_{1}(t) & \leq\left|z_{1}\right|\left|d_{1}(t)\right| \leq\left|z_{1}\right| W_{1}=\left|z_{1}\right|\left(\hat{W}_{1}+\hat{W}_{1}\right) \\
& \leq \frac{z_{1}^{2} \hat{W}_{1}^{2}}{\sqrt{z_{1}^{2} \hat{W}_{1}^{2}+\sigma(t)^{2}}}+\sigma(t)+\left|z_{1}\right| \widetilde{W}_{1} .
\end{aligned}
$$

On designing the virtual control law $\alpha_{1}$ as

$$
\alpha_{1}=-k_{1} z_{1}+\dot{y}_{r}-\hat{\varepsilon}_{1} \frac{z_{1} D_{1}^{2}}{\sqrt{z_{1}^{2} D_{1}^{2}+\sigma(t)^{2}}}-\frac{z_{1} \hat{W}_{1}^{2}}{\sqrt{z_{1}^{2} \hat{W}_{1}^{2}+\sigma(t)^{2}}},
$$

the update laws of $\hat{\varepsilon}_{1}$ and $\hat{W}_{1}$ can be designed as

$$
\begin{aligned}
& \dot{\hat{\varepsilon}}_{1}=\gamma_{1} \frac{z_{1}^{2} D_{1}^{2}}{\sqrt{z_{1}^{2} D_{1}^{2}+\sigma(t)^{2}}}, \\
& \dot{\hat{W}}_{1}=\beta_{1}\left|z_{1}\right| .
\end{aligned}
$$

In view of (5)-(11), one has

$$
\begin{aligned}
\dot{V}_{1}= & z_{1}\left(x_{2}+\theta_{1} f_{1, \varrho(t)} \overline{\left(x_{1}\right)}+d_{1}(t)-\dot{y}_{r}+\left(-k_{1} z_{1}+\dot{y}_{r}\right.\right. \\
& \left.\left.-\hat{\varepsilon}_{1} \frac{z_{1} D_{1}^{2}}{\sqrt{z_{1}^{2} D_{1}^{2}+\sigma(t)^{2}}}-\frac{z_{1} \widehat{W}_{1}^{2}}{\sqrt{z_{1}^{2} \widehat{W}_{1}^{2}+\sigma(t)^{2}}}\right)-\alpha_{1}\right) \\
& -\frac{1}{\gamma_{1}} \widetilde{\varepsilon}_{1}\left(\gamma_{1} \frac{z_{1}^{2} D_{1}^{2}}{\sqrt{z_{1}^{2} D_{1}^{2}+\sigma(t)^{2}}}\right)-\frac{1}{\beta_{1}} \hat{W}_{1}\left(\beta_{1}\left|z_{1}\right|\right) \\
\leq & -k_{1} z_{1}^{2}+\left(x_{2}-\alpha_{1}\right) z_{1}+\left(1+\varepsilon_{1}\right) \sigma(t) .
\end{aligned}
$$

Design the second error signal in the backstepping as $x_{2}-\alpha_{1}$ such that it can avoid the "explosion of complexity" problem. Let $\alpha_{1}$ pass through the following novel nonlinear filter to obtain a filtered virtual controller $s_{1}$ :

$$
\begin{aligned}
& \tau_{1} \dot{s}_{1}=-e_{1}-\frac{\tau_{1} \hat{M}_{1}^{2} e_{1}}{\sqrt{\widehat{M}_{1}^{2} e_{1}^{2}+\sigma^{2}(t)}}-\tau_{1} z_{1}, \\
& s_{1}(0)=\alpha_{1}(0),
\end{aligned}
$$

where $e_{1}=s_{1}-\alpha_{1}$ is the first boundary layer error, $\tau_{1}>0$ is a design parameter, $\widehat{M}_{1}$ is the estimate of $M_{1}$ and it will be clarified later, and $\sigma(t)$ is any positive uniform continuous and bounded function, which satisfies

$$
\begin{aligned}
\lim _{t \rightarrow \infty} \int_{0}^{t} \sigma(\tau) d \tau & \leq \sigma_{1}<+\infty, \\
|\dot{\sigma}(t)| & \leq \sigma_{2}<+\infty,
\end{aligned}
$$

where $\sigma_{1}$ and $\sigma_{2}$ are any positive constants.

Remark 1. In this paper, the nonlinear filter (13) is introduced to construct the desired controller. Compared with the reported results on adaptive DSC with a linear low-pass filter (e.g., see [30-37]), the advantage of our deign is that the asymptotic tracking control performance can be guaranteed, and the stability analysis can be completed successfully under the proposed controller.

Step $i(i=2, \ldots, n-1)$. The $i$ th surface error is designed as $z_{i}=x_{i}-s_{i-1}$; then, we get the following equation:

$$
\dot{z}_{i}=x_{i+1}+\theta_{i} f_{i, \varrho(t)}+d_{i}(t)+\frac{\widehat{M}_{i-1}^{2} e_{i-1}}{\sqrt{\widehat{M}_{i-1}^{2} e_{i-1}^{2}+\sigma^{2}(t)}}+z_{i-1}+\frac{e_{i-1}}{\tau_{i-1}} \text {. }
$$

Design the virtual control law $\alpha_{i}$ and the update laws $\widehat{\varepsilon}_{i}$ and $\widehat{W}_{i}$ as follows:

$$
\begin{aligned}
& \alpha_{i}=-k_{i} z_{i}-2 z_{i-1}-\hat{\varepsilon}_{i} \frac{z_{i} D_{i}^{2}}{\sqrt{z_{i}^{2} D_{i}^{2}+\sigma^{2}(t)}} \\
& -\frac{z_{i} \hat{W}_{i}^{2}}{\sqrt{z_{i}^{2} \hat{W}_{i}^{2}+\sigma^{2}(t)}}-\frac{\hat{M}_{i-1}^{2} e_{i-1}}{\sqrt{\hat{M}_{i-1}^{2} e_{i-1}^{2}+\sigma^{2}(t)}}-\frac{e_{i-1}}{\tau_{i-1}}, \\
& \dot{\hat{\varepsilon}}_{1}=\gamma_{i} \frac{z_{i}^{2} D_{i}^{2}}{\sqrt{z_{i}^{2} D_{i}^{2}+\sigma^{2}(t)}}, \\
& \dot{\hat{W}}_{1}=\beta_{i}\left|z_{i}\right|,
\end{aligned}
$$

where $k_{i}, \gamma_{i}$, and $\beta_{i}$ are the positive design parameters.

Design the Lyapunov function candidate $V_{i}$ as

$$
V_{i}=V_{i-1}+\frac{1}{2} z_{i}^{2}+\frac{1}{2 \gamma_{i}} \widetilde{\varepsilon}_{i}^{2}+\frac{1}{2 \beta_{i}} \widetilde{W}_{i}^{2},
$$

where $\gamma_{i}$ and $\beta_{i}$ are the positive design parameters. 
In view of equations (15)-(19), consider the time derivative of $V_{i}$ as

$$
\begin{aligned}
\dot{V}_{i} & =\dot{V}_{i-1}+z_{i} \dot{z}_{i}-\frac{1}{\gamma_{i}} \widetilde{\varepsilon}_{i} \dot{\hat{\varepsilon}}_{i}-\frac{1}{\beta_{i}} \widetilde{W}_{i} \dot{\hat{W}}_{i} \\
& \leq-\sum_{j=1}^{i} k_{j} z_{j}^{2}+\sum_{j=1}^{i-1} z_{j} e_{j}+\left(x_{i+1}-\alpha_{i}\right) z_{i}+\left(i+\sum_{j=1}^{i} \varepsilon_{j}\right) \sigma(t) .
\end{aligned}
$$

Let $\alpha_{i}$ pass through the following nonlinear filter to obtain a filtered virtual controller $s_{i}$

$$
\begin{aligned}
\tau_{i} \dot{s}_{i} & =-e_{i}-\frac{\tau_{i} \hat{M}_{i}^{2} e_{i}}{\sqrt{\hat{M}_{i}^{2} e_{i}^{2}+\sigma^{2}(t)}}-\tau_{i} z_{i}, \\
s_{i}(0) & =\alpha_{i}(0),
\end{aligned}
$$

and define

$$
e_{i}=s_{i}-\alpha_{i}
$$

where $e_{i}$ means the $i$ th boundary layer error and $\tau_{i}$ is a filter time constant.

Step $n$. Considering the $n$th surface error as $z_{n}=x_{n}-s_{n-1}$, one has

$$
\dot{z}_{n}=u+\theta_{n} f_{n, \varrho(t)}+d_{n}(t)+\frac{\widehat{M}_{n-1}^{2} e_{n-1}}{\sqrt{\widehat{M}_{n-1}^{2} e_{n-1}^{2}+\sigma^{2}(t)}}+z_{n-1}+\frac{e_{n-1}}{\tau_{n-1}} .
$$

Design the actual control law $u$ as

$$
\begin{aligned}
u= & -k_{n} z_{n}-2 z_{n-1}-\hat{\varepsilon}_{n} \frac{z_{n} D_{n}^{2}}{\sqrt{z_{n}^{2} D_{n}^{2}+\sigma^{2}(t)}} \\
& -\frac{z_{n} \hat{W}_{n}^{2}}{\sqrt{z_{n}^{2} \hat{W}_{n}^{2}+\sigma^{2}(t)}}-\frac{\hat{M}_{n-1}^{2} e_{n-1}}{\sqrt{\hat{M}_{n-1}^{2} e_{n-1}^{2}+\sigma^{2}(t)}}-\frac{e_{n-1}}{\tau_{n-1}},
\end{aligned}
$$

and consider the update laws for $\widehat{\varepsilon}_{i}$ and $\widehat{W}_{n}$ as follows:

$$
\begin{aligned}
& \dot{\hat{\varepsilon}}_{n}=\gamma_{n} \frac{z_{n}^{2} D_{n}^{2}}{\sqrt{z_{n}^{2} D_{n}^{2}+\sigma^{2}(t)}}, \\
& \dot{\hat{W}}_{n}=\beta_{n}\left|z_{n}\right|,
\end{aligned}
$$

where $k_{n}, \beta_{n}$, and $\gamma_{n}$ are the positive design parameters.

Design the Lyapunov function candidate $V_{n}$ as follows:

$$
V_{n}=V_{n-1}+\frac{1}{2} z_{n}^{2}+\frac{1}{2 \gamma_{n}} \widetilde{\varepsilon}_{n}^{2}+\frac{1}{2 \beta_{n}} \tilde{W}_{n}^{2}
$$

where $\gamma_{n}$ and $\beta_{n}$ are the positive design parameters.

Then, considering equations (23)-(27), we have

$$
\begin{aligned}
\dot{V}_{n} & =\dot{V}_{n-1}+z_{n} \dot{z}_{n}-\frac{1}{\gamma_{n}} \widetilde{\varepsilon}_{n} \dot{\widehat{\varepsilon}}_{n}-\frac{1}{\beta_{n}} \widetilde{W}_{n} \dot{\hat{W}}_{n} \\
& \leq-\sum_{j=1}^{n} k_{j} z_{j}^{2}+\sum_{j=1}^{n-1} z_{j} e_{j}+\left(n+\sum_{j=1}^{n} \varepsilon_{j}\right) \sigma(t) .
\end{aligned}
$$

2.3. Stability Analysis. Based on inequality (28), the main result of this paper is presented by the following theorem.

Theorem 1. Consider the closed-loop system consisting of the plant (1), the nonlinear filters (13), (22), the actual controller (24), and the adaptive laws (10), (11), (17), (18), (25), and(26). Suppose that Assumptions 1-2 hold, for any initial conditions satisfying $V(0) \leq q$, where $q$ is a given constant, there exit design parameters $k_{i}, \beta_{i}, \gamma_{i}, i=1, \ldots, n, \tau_{j}$, and $\eta_{j}$, $j=1, \ldots, n-1$, such that the following statements hold:

(i) All the resulting closed-loop signals are semiglobally bounded

(ii) The tracking error $z_{1}=y-y_{r}$ converges to zero asymptotically

Proof. The compact sets are defined as

$$
\begin{aligned}
& \Omega_{1}=\left\{\left[y_{r}, \dot{y}_{r}, \ddot{y}_{r}\right]^{T}: y_{r}^{2}+\dot{y}_{r}^{2}+\ddot{y}_{r}^{2} \leq B_{0}\right\}, \\
& \Omega_{2}=\{V(t) \leq q\},
\end{aligned}
$$

where $B_{0}$ is a known positive constant. Note that set $\Omega_{1} \times \Omega_{2}$ is also a compact in $R^{4 n+1} . \sigma(t)$ and $\dot{\sigma}(t)$ are the bounded functions.

Differentiating the boundary layer errors $e_{i}=s_{i}-\alpha_{i}$ yields

$$
\begin{aligned}
\dot{e}_{i}= & \frac{-e_{i}}{\tau_{i}}-\frac{\hat{M}_{i}^{2} e_{i}}{\sqrt{\hat{M}_{i}^{2} e_{i}^{2}+\sigma^{2}(t)}}-z_{i}+B_{i}\left(z_{1}, \ldots, z_{i+1}, e_{1}, \ldots,\right. \\
& e_{i}, \hat{\varepsilon}_{1}, \ldots, \hat{\varepsilon}_{i}, \hat{M}_{1}, \ldots, \hat{M}_{i}, \hat{W}_{1}, \ldots, \hat{W}_{i}, y_{r}, \ddot{y}_{r}, \ddot{y}_{r}, \sigma(t), \\
& \dot{\sigma}(t)), \quad i=1, \ldots, n-1,
\end{aligned}
$$

where

$$
\begin{aligned}
B_{1}(\cdot)= & -\dot{\alpha}_{1} \\
= & -\frac{\partial \alpha_{1}}{\partial x_{1}} \dot{x}_{1}-\frac{\partial \alpha_{1}}{\partial \widehat{\varepsilon}_{1}} \dot{\hat{\varepsilon}}_{1}-\frac{\partial \alpha_{1}}{\partial y_{r}} \dot{y}_{r}-\frac{\partial \alpha_{1}}{\partial \dot{y}_{r}} \ddot{y}_{r}-\frac{\partial \alpha_{1}}{\partial \widehat{W}_{1}} \dot{\hat{W}}_{1}, \\
B_{i}(\cdot)= & -\dot{\alpha}_{i} \\
= & -\sum_{j=1}^{i} \frac{\partial \alpha_{i}}{\partial x_{j}} \dot{x}_{j}-\frac{\partial \alpha_{i}}{\partial \widehat{\hat{\varepsilon}}_{j}}-\frac{\partial \alpha_{i}}{\partial e_{i-1}} \dot{e}_{i-1} \\
& -\frac{\partial \alpha_{i}}{\partial \hat{M}_{i-1}} \dot{\hat{M}}_{i-1}-\frac{\partial \alpha_{i}}{\partial \hat{W}_{i}} \dot{\hat{W}}_{i}-\frac{\partial \alpha_{i}}{\partial y_{r}} \dot{y}_{r}-\frac{\partial \alpha_{i}}{\partial \dot{y}_{r}} \ddot{y}_{r}-\frac{\partial \alpha_{i}}{\partial \sigma(t)} \dot{\sigma}(t),
\end{aligned}
$$




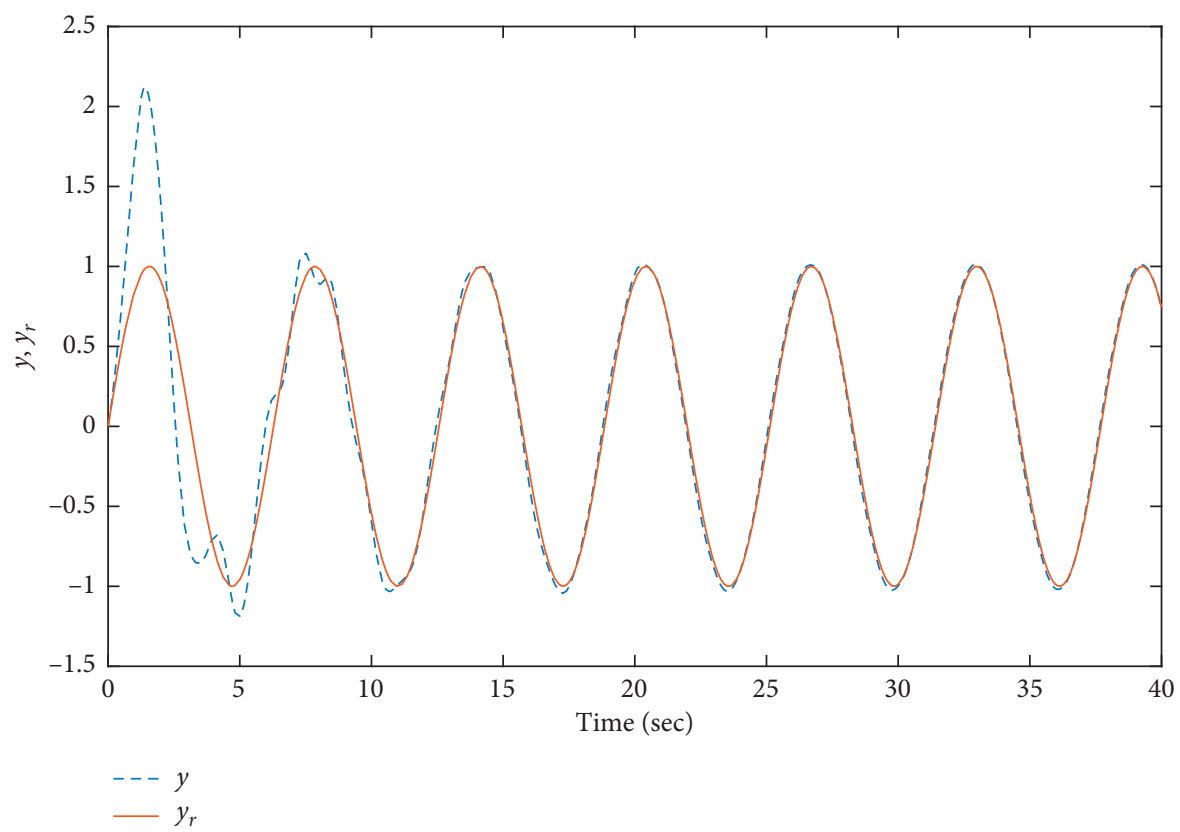

FIGURE 1: The reference signal $y_{r}$ and the output signal $y$.

are the continuous functions on $\Omega_{1} \times \Omega_{2}$. As a consequence, a positive constant $M_{i}$ can be obtained such that $\left|B_{i}(\cdot)\right| \leq M_{i}$, where $M_{i}>0$ is an unknown constant.

We consider the Lyapunov function candidate as follows:

$$
V=V_{n}+\sum_{i=1}^{n-1} \frac{1}{2} e_{i}^{2}+\sum_{i=1}^{n-1} \frac{1}{2 \eta_{i}} \tilde{M}_{i}^{2}
$$

where $\eta_{i}, i=1, \ldots, n-1$ are the positive design parameters.

The time derivative of $V$ is

$$
\begin{aligned}
\dot{V}= & \dot{V}_{n}+\sum_{i=1}^{n-1} e_{i} \dot{e}_{i}-\sum_{i=1}^{n-1} \frac{1}{\eta_{i}} \widetilde{M}_{i} \dot{\hat{M}}_{i} \\
\leq & -\sum_{i=1}^{n} k_{i} z_{i}^{2}-\sum_{i=1}^{n-1} \frac{e_{i}^{2}}{\tau_{i}}-\sum_{i=1}^{n-1} \frac{\hat{M}_{i}^{2} e_{i}}{\sqrt{\hat{M}_{i}^{2} e_{i}^{2}+\sigma^{2}(t)}} \\
& +\sum_{i=1}^{n-1} M_{i}\left|e_{i}\right|-\sum_{i=1}^{n-1} \frac{1}{\eta_{i}} \tilde{M}_{i} \dot{\hat{M}}_{i}+\left(n+\sum_{j=1}^{n} \varepsilon_{j}\right) \sigma(t) .
\end{aligned}
$$
$1:$

We can obtain the following inequality in view of Lemma

$$
\begin{aligned}
M_{i}\left|e_{i}\right| & =\hat{M}_{i}\left|e_{i}\right|+\hat{M}_{i}\left|e_{i}\right| \\
& \leq \frac{\hat{M}_{i}^{2} e_{i}^{2}}{\sqrt{\hat{M}_{i}^{2} e_{i}^{2}+\sigma^{2}(t)}}+\sigma(t)+\tilde{M}_{i}\left|e_{i}\right|,
\end{aligned}
$$

and then, we have

$$
\dot{V} \leq-\sum_{i=1}^{n} k_{i} z_{i}^{2}-\sum_{i=1}^{n-1} \frac{e_{i}^{2}}{\tau_{i}}-\sum_{i=1}^{n-1} \frac{1}{\eta_{i}} \tilde{M}_{i}\left(\dot{\hat{M}}_{i}-\beta_{i}\left|e_{i}\right|\right)+\Phi \sigma(t),
$$

where $₫:=2 n+\sum_{j=1}^{n} \varepsilon_{j}-1$ is a constant.

Consider the update laws for $\widehat{M}_{i}$ as

$$
\dot{\hat{M}}_{i}=\eta_{i}\left|e_{i}\right|, \quad i=1, \ldots, n-1 \text {. }
$$

From equation (35), the following inequality can be obtained:

$$
\dot{V} \leq-\sum_{i=1}^{n} k_{i} z_{i}^{2}-\sum_{i=1}^{n-1} \frac{e_{i}^{2}}{\tau_{i}}+\omega \sigma(t) .
$$

Integrating inequality (37) over $[0, t]$ yields

$$
\begin{aligned}
V(t) & \leq V(0)-\int_{0}^{t}\left(\sum_{i=1}^{n} k_{i} z_{i}^{2}(\kappa)+\sum_{i=1}^{n} \frac{e_{i}^{2}(\kappa)}{\tau_{i}}\right) d \kappa+\omega \int_{0}^{t} \sigma(\kappa) d \kappa \\
& \leq V(0)+\Phi \sigma_{1},
\end{aligned}
$$

which means that $z_{i}, z_{n}, \widetilde{\varepsilon}_{i}, \widehat{\varepsilon}_{n}, \widehat{W}_{i}, \widehat{W}_{n}, e_{i}$, and $\widehat{M}_{i}$ $, i=1, \ldots, n-1$ are bounded. Therefore, we also conclude that $x_{i}, x_{n}, s_{i}, \alpha_{i}$, and $u, i=1, \ldots, n-1$ are bounded. From inequality (38), we can obtain the following inequality:

$$
\int_{0}^{t} \sum_{i=1}^{n} k_{i} z_{i}^{2}(\kappa) d \kappa \leq V(0)+\varpi \sigma_{1}
$$

By applying Barbalat's lemma [40, 41] to inequality (39), it is concluded that

$$
\lim _{t \rightarrow \infty} z_{1}=0
$$

that is to say, the asymptotic stability is accomplished.

Remark 2. For uncertain switched systems (e.g., see [2123]), lots of adaptive control schemes have been developed by the conventional backstepping method. This paper 


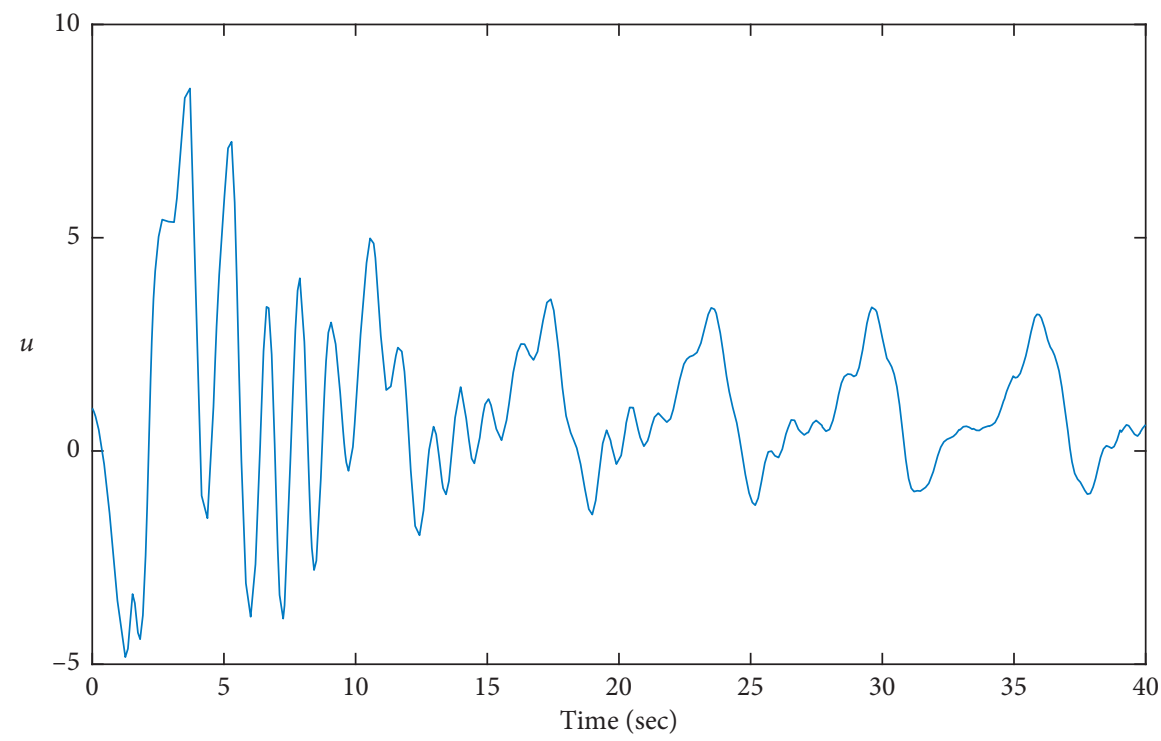

Figure 2: The control signal $u$.

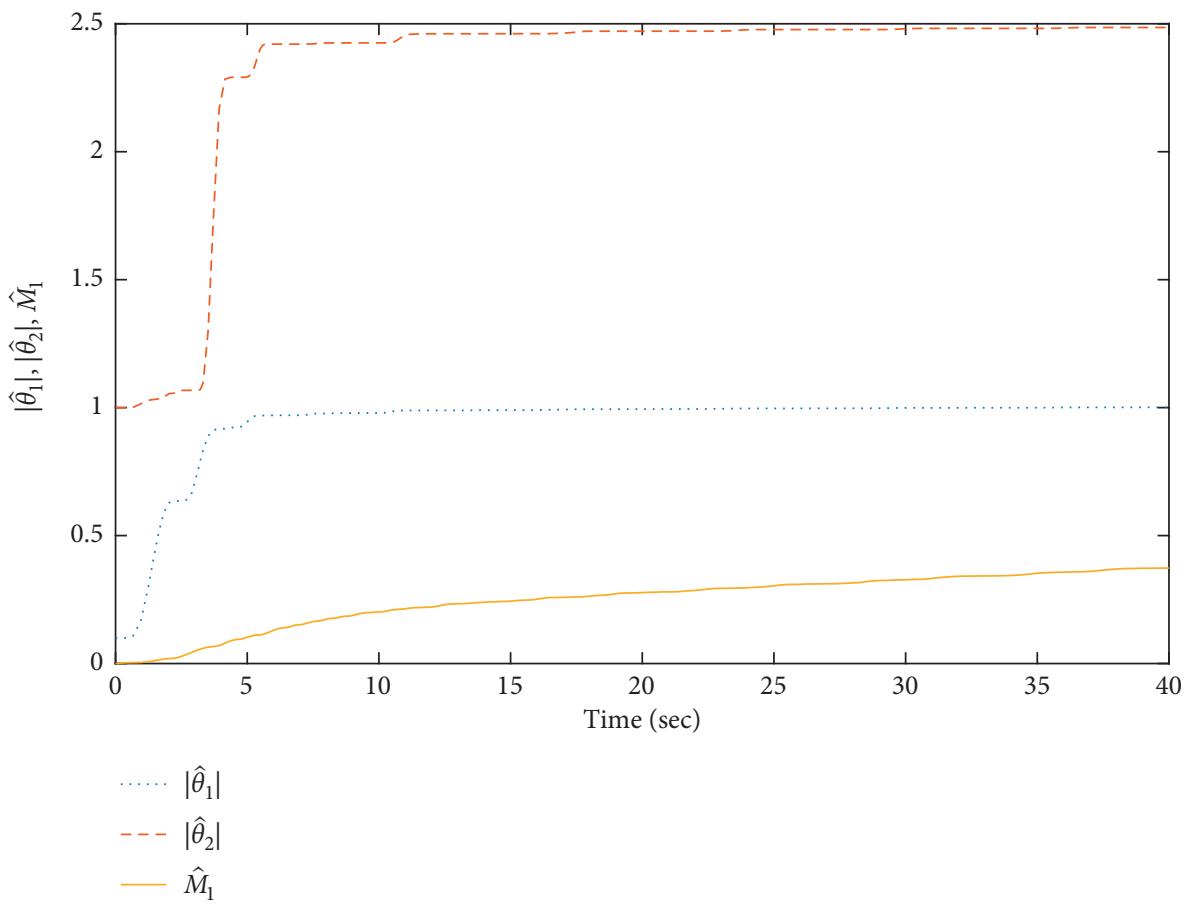

Figure 3: Adaptive parameters $\left|\widehat{\theta}_{1}\right|,\left|\widehat{\theta}_{2}\right|$, and $\widehat{M}_{1}$.

introduces the adaptive DSC method to solve the "explosion of complexity" problem existing in the aforementioned literature studies, and the asymptotic tracking performance has been achieved under the developed adaptive controller.

Remark 3. Recently, some novel adaptive control schemes have been proposed for uncertain systems with time-varying performance bounds and actuator failures in $[42,43]$, where the "explosion of complexity" problem cannot be well solved and the asymptotic tracking performance is not achieved. Of course, this paper does not consider the actuator failure phenomenon of the controlled system, which is the further study program in the future.

\section{Simulation Example}

Consider the following nonlinear switched system:

$$
\left\{\begin{array}{l}
\dot{x}_{1}=x_{2}+\theta_{1} f_{1, \varrho(t)} \overline{\left(x_{1}\right)}+d_{1}(t), \\
\dot{x}_{2}=u+\theta_{2} f_{2, \varrho(t)} \overline{\left(x_{2}\right)}+d_{2}(t), \\
y=x_{1},
\end{array}\right.
$$




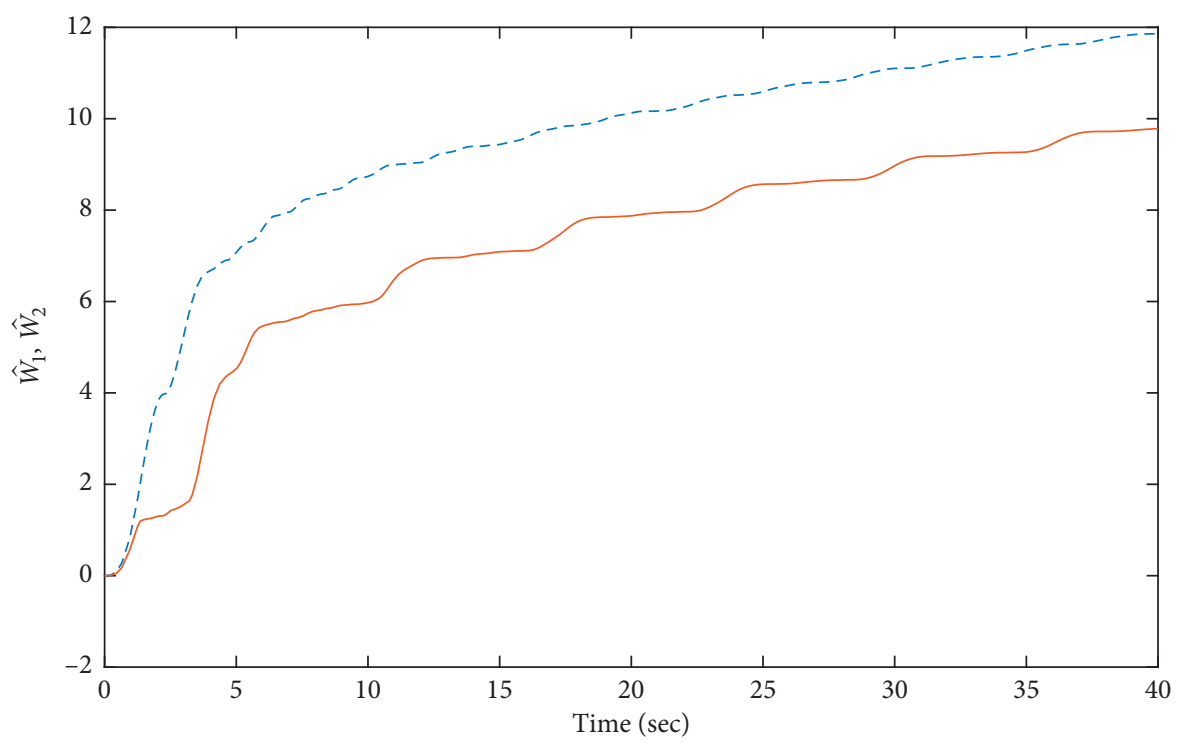

$\begin{array}{ll}-- & \hat{W}_{1} \\ - & \hat{W}_{2}\end{array}$

FIGURE 4: Adaptive parameters $\widehat{W}_{1}$ and $\widehat{W}_{2}$.

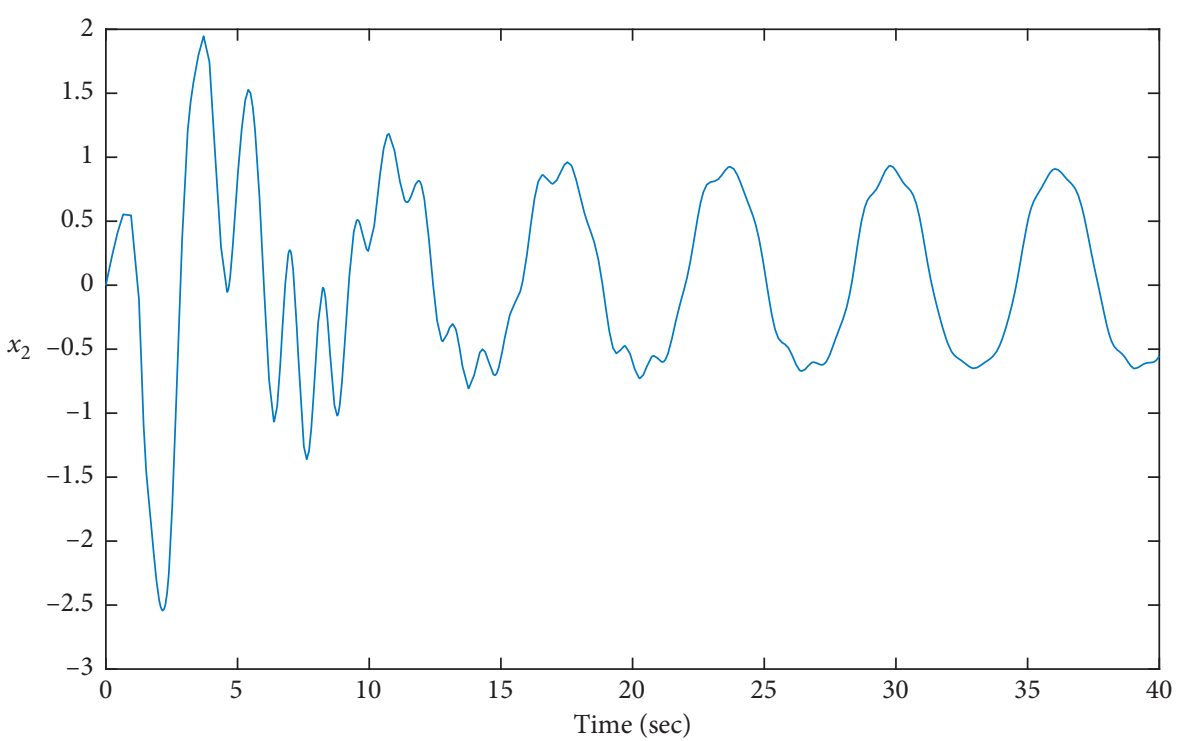

Figure 5: State $x_{2}$.

where $\theta_{1}$ and $\theta_{2}$ are the unknown parameters; $d_{1}(t)=\cos (t)$ and $d_{2}(t)=\sin (t) ; \varrho(t) \in\{1,2\}$; and the switched functions are designed as follows: $f_{1,1}=x_{1} e^{-0.5 x_{1}}, f_{1,2}=\sin \left(x_{1}\right)$, $f_{2,1}=\sin \left(x_{1} x_{2}\right)$, and $f_{2,2}=x_{1} x_{2}^{2}$. When the output $y$ satisfies $\quad y>0, \quad f_{i, \sigma(t)}=f_{i, 1}, i=1,2$. Otherwise, $f_{i, \sigma(t)}=f_{i, 2}, i=1,2$. For simulation purpose, it is assumed that $\theta_{1}=1$ and $\theta_{2}=2.5$. The objective of control is that the output $y(t)$ asymptotically tracks the desired trajectory $y_{r}(t)=\sin (t)$ via the proposed adaptive controller $u$.

The design parameters are selected as $k_{1}=1.2, k_{2}=2$, $\gamma_{1}=1.12, \quad \gamma_{2}=1.02, \quad \beta_{1}=\beta_{2}=3, \eta_{1}=0.25, \quad \tau_{1}=1$, and $\sigma(t)=3 e^{-0.0001 t}$. The initial conditions of this switched system are chosen as $\left[x_{1}(0), x_{2}(0)\right]^{T}=[0,0]^{T}, \hat{\varepsilon}_{1}(0)=0.1$, $\widehat{\varepsilon}_{2}(0)=1, \hat{W}_{1}(0)=\hat{W}_{2}(0)=0$, and $\hat{M}_{1}(0)=0$. The simulation results are displayed in Figures 1-5. Figure 1 shows the output tracking performance under the presented DSC scheme, from which it can be analyzed that the asymptotic output tracking has been achieved. Figure 2 shows the control signal $u$. Figures 3 and 4 show the adaptive parameters. The state $x_{2}$ is shown in Figure 5. The switching signal is presented in Figure 6. All these simulation results show that the closed-loop signals are bounded. 


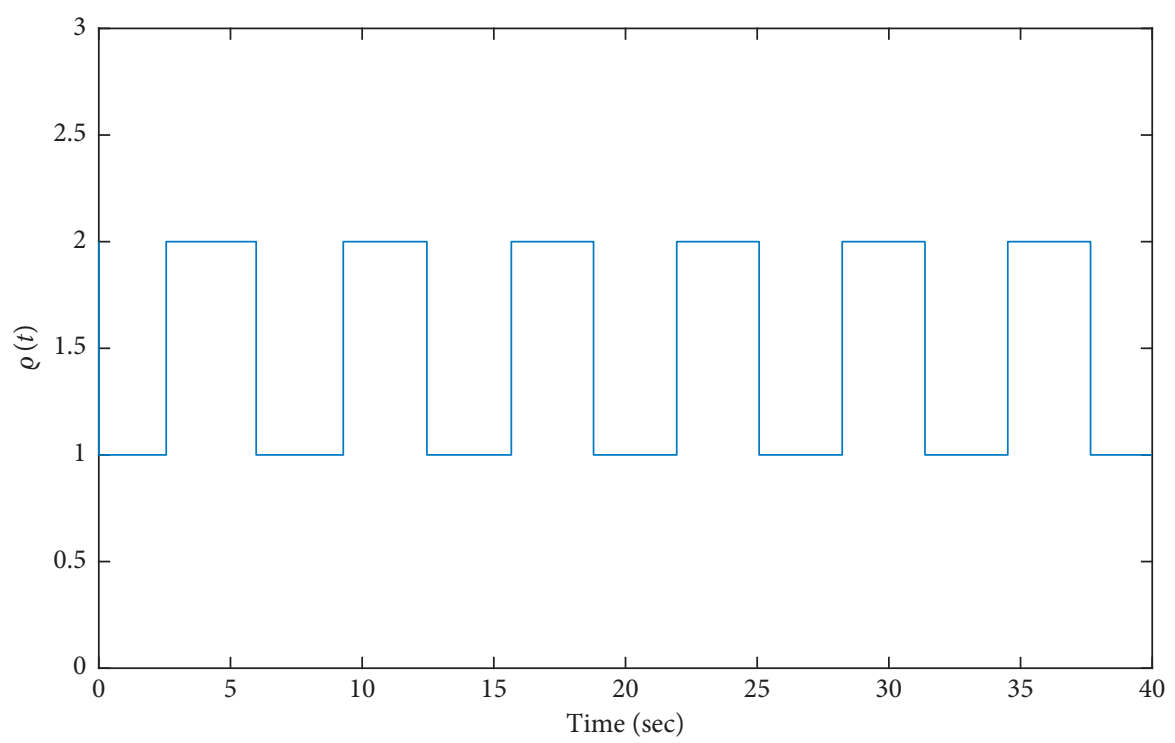

FIgURE 6: Switching signal $\varrho(t)$.

\section{Conclusions}

This paper studies the adaptive tracking control problem for a class of uncertain nonlinear systems with perturbations and switching signal. To deal with the switched signals of the considered system, the common Lyapunov function method is employed to deign the desired controller. Furthermore, according to a nonlinear filter, a DSC method is presented to overcome the "explosion of complexity" problem. Under the proposed controller, it has been expressed that all the closedloop signals remain semiglobally bounded, and the tracking error converges to zero asymptotically. In the end of this paper, to checkout the validity of the control scheme, a simulation example is presented.

\section{Data Availability}

This paper is a theoretical study and no data were used to support this study.

\section{Conflicts of Interest}

The authors declare that they have no conflict of interest.

\section{Acknowledgments}

This work was supported by the National Natural Science Foundation of China (Nos. 61603003, 61702012, and 60973050), the Natural Science Foundation of Anhui Province (1608085QF131), the China Postdoctoral Science Foundation (2017M620245), the Anhui Province Department of Education University Scientific Research Fund (KJ2018A0359 and KJ2019A0570), the Foundation of the University Research and Innovation Platform Team for Intelligent Perception and Computing of Anhui Province, the Program for Academic Top-Notch Talents of University Disciplines (gxbjZD21), and the Program for Innovative Research Team in Anqing Normal University.

\section{References}

[1] C. Briat, "Convex lifted conditions for robust -stability analysis and -stabilization of linear discrete-time switched systems with minimum dwell-time constraint," Automatica, vol. 50, no. 3, pp. 976-983, 2014.

[2] D. Zhang, P. Shi, W.-A. Zhang, and L. Yu, "Energy-efficient distributed filtering in sensor networks: a unified switched system approach," IEEE Transactions on Cybernetics, vol. 47, no. 7, pp. 1618-1629, 2017.

[3] W. Xiang and J. Xiao, "Stabilization of switched continuoustime systems with all modes unstable via dwell time switching," Automatica, vol. 50, no. 3, pp. 940-945, 2014.

[4] W. W. Xie, C. C. Wen, and Z. Z. Li, "Input-to-state stabilization of switched nonlinear systems," IEEE Transactions on Automatic Control, vol. 46, no. 7, pp. 1111-1116, 2001.

[5] L. Zhang, S. Zhuang, and P. Shi, "Non-weighted quasi-timedependent $H_{\infty}$ filtering for switched linear systems with persistent dwell-time," Automatica, vol. 54, no. 6, pp. 201-209, 2015.

[6] X. Zhao, S. Yin, H. Li, and B. Niu, "Switching stabilization for a class of slowly switched systems," IEEE Transactions on Automatic Control, vol. 60, no. 1, pp. 221-226, 2015.

[7] P. Colaneri, R. H. Middleton, Z. Chen, D. Caporale, and F. Blanchini, "Convexity of the cost functional in an optimal control problem for a class of positive switched systems," Automatica, vol. 50, no. 4, pp. 1227-1234, 2014.

[8] X. Zhao, L. Zhang, P. Shi, and M. Liu, "Stability and stabilization of switched linear systems with mode-dependent average dwell time," IEEE Transactions on Automatic Control, vol. 57, no. 7, pp. 1809-1815, 2012.

[9] D. D. Wang, W. W. Wang, and P. P. Shi, "Robust fault detection for switched linear systems with state delays," IEEE Transactions on Systems, Man, and Cybernetics, Part B (Cybernetics), vol. 39, no. 3, pp. 800-805, 2009.

[10] J. Xiong, J. Lam, H. Gao, D. W. C. Ho, and C. Ho, "On robust stabilization of markovian jump systems with uncertain switching probabilities," Automatica, vol. 41, no. 5, pp. 897-903, 2005.

[11] L. L. Zhang and P. P. Shi, "Stability, $\$\{1\} \_\{2\} \$-G a i n$ and asynchronous $\$\{\mathrm{H}\} \_\{\{\backslash \text { infty }\}\}$ control of discrete-time switched systems with average dwell time," IEEE Transactions On Automatic Control, vol. 54, no. 9, pp. 2192-2199, 2009. 
[12] M. Wang, J. Qiu, M. Chadli, and M. Wang, "A switched system approach to exponential stabilization of sampled-data T-S fuzzy systems with packet dropouts," IEEE Transactions on Cybernetics, vol. 46, no. 12, pp. 3145-3156, 2016.

[13] P. Shi, X. Su, and F. Li, "Dissipativity-based filtering for fuzzy switched systems with stochastic perturbation," IEEE Transactions on Automatic Control, vol. 61, no. 6, pp. 1694-1699, 2016.

[14] X. Zhao, P. Shi, Y. Yin, and S. K. Nguang, "New results on stability of slowly switched systems: a multiple discontinuous lyapunov function approach," IEEE Transactions on Automatic Control, vol. 62, no. 7, pp. 3502-3509, 2017.

[15] M. Toulabi, A. S. Dobakhshari, and A. M. Ranjbar, "An adaptive feedback linearization approach to inertial frequency response of wind turbines," IEEE Transactions on Sustainable Energy, vol. 8, no. 3, pp. 916-926, 2017.

[16] C. L. P. Chen, G.-X. Wen, Y.-J. Liu, and Z. Liu, "Observerbased adaptive backstepping consensus tracking control for high-order nonlinear semi-strict-feedback multiagent systems," IEEE Transactions on Cybernetics, vol. 46, no. 7, pp. 1591-1601, 2016.

[17] Z. Liu, X. Tan, R. Yuan, G. Fan, and J. Yi, "Immersion and invariance-based output feedback control of air-breathing hypersonic vehicles," IEEE Transactions on Automation Science and Engineering, vol. 13, no. 1, pp. 394-402, 2016.

[18] H. Li, C. Wu, X. Jing, and L. Wu, "Fuzzy tracking control for nonlinear networked systems," IEEE Transactions on Cybernetics, vol. 47, no. 8, pp. 2020-2031, 2017.

[19] J. Wu, J. Li, and W. Chen, "Practical adaptive fuzzy tracking control for a class of perturbed nonlinear systems with backlash nonlinearity," Information Sciences, vol. 420, pp. 517-531, 2017.

[20] J. Wu, J. Li, G. Zong, and W. Chen, "Global finite-time adaptive stabilization of nonlinearly parametrized systems with multiple unknown control directions," IEEE Transactions on Systems, Man, and Cybernetics: Systems, vol. 47, no. 7, pp. 1405-1414, 2017.

[21] X. Zhao, P. Shi, and X. Zheng, "Fuzzy adaptive control design and discretization for a class of nonlinear uncertain systems," IEEE Transactions on Cybernetics, vol. 46, no. 6, pp. 1476$1483,2016$.

[22] X. Zhao, X. Zheng, B. Niu, and L. Liu, "Adaptive tracking control for a class of uncertain switched nonlinear systems," Automatica, vol. 52, no. 2, pp. 185-191, 2015.

[23] Y. Li, S. Sui, and S. Tong, "Adaptive fuzzy control design for stochastic nonlinear switched systems with arbitrary switchings and unmodeled dynamics," IEEE Transactions on Cybernetics, vol. 47, no. 2, pp. 403-414, 2017.

[24] M. Chen and G. Tao, "Adaptive fault-tolerant control of uncertain nonlinear large-scale systems with unknown dead zone," IEEE Transactions on Cybernetics, vol. 46, no. 8, pp. 1851-1862, 2016.

[25] H. Wang, P. X. Liu, and B Niu, "Robust fuzzy adaptive tracking control for nonaffine stochastic nonlinear switching systems," IEEE Transactions on Cybernetics, vol. 48, no. 8, pp. 2462-2471, 2018.

[26] J. Huang, W. Wang, C. Wen, and J. Zhou, “Adaptive control of a class of strict-feedback time-varying nonlinear systems with unknown control coefficients," Automatica, vol. 93, pp. $98-105,2018$.

[27] J. Huang and Q.-G. Wang, "Decentralized adaptive control of interconnected nonlinear systems with unknown control directions," ISA Transaction, vol. 74, pp. 60-66, 2018.

[28] J. Huang, W. Wang, C. Wen, and G. Li, "Adaptive eventtriggered control of nonlinear systems with controller and parameter estimator triggering," IEEE Transactions on Automatic Control, 2019.

[29] D. Zhai, C. Xi, L. An, J. Dong, and Q. Zhang, "Prescribed performance switched adaptive dynamic surface control of switched nonlinear systems with average dwell time," IEEE Transactions on Systems, Man, and Cybernetics: Systems, vol. 47, no. 7, pp. 1257-1269, 2017.

[30] M. Chen, G. Tao, and B. Jiang, "Dynamic surface control using neural networks for a class of uncertain nonlinear systems with input saturation," IEEE Transactions on Neural Networks and Learning Systems, vol. 26, no. 9, pp. 2086-2097, 2015.

[31] J. Chen, Z. Li, G. Zhang, and M. Gan, "Adaptive robust dynamic surface control with composite adaptation laws," International Journal of Adaptive Control and Signal Processing, vol. 24, no. 12, pp. 1036-1050, 2010.

[32] M. Gan, J. Chen, and Z. Li, "Multiple-model adaptive robust dynamic surface control with estimator resetting," International Journal of Adaptive Control and Signal Processing, vol. 29, no. 8, pp. 939-953, 2015.

[33] Y. Pan and H. Yu, "Composite learning from adaptive dynamic surface control," IEEE Transactions on Automatic Control, vol. 61, no. 9, pp. 2603-2609, 2016.

[34] B. Xu, C. Yang, and Y. Pan, "Global neural dynamic surface tracking control of strict-feedback systems with application to hypersonic flight vehicle," IEEE Transactions on Neural Networks and Learning Systems, vol. 26, no. 10, pp. 25632575, 2015.

[35] X. Zhang, C.-Y. Su, Y. Lin, L. Ma, and J. Wang, "Adaptive neural network dynamic surface control for a class of timedelay nonlinear systems with hysteresis inputs and dynamic uncertainties," IEEE Transactions on Neural Networks and Learning Systems, vol. 26, no. 11, pp. 2844-2860, 2015.

[36] X. Shi, C.-C. Lim, P. Shi, and S. Xu, "Adaptive neural dynamic surface control for nonstrict-feedback systems with output dead zone," IEEE Transactions on Neural Networks and Learning Systems, vol. 29, no. 11, pp. 5200-5213, 2018.

[37] Z. Liu, X. Dong, J. Xue, H. Li, and Y. Chen, "Adaptive neural control for a class of pure-feedback nonlinear systems via dynamic surface technique," IEEE Transactions on Neural Networks and Learning Systems, vol. 27, no. 9, pp. 1969-1975, 2016.

[38] Y.-H. Liu, "Adaptive dynamic surface asymptotic tracking for a class of uncertain nonlinear systems," International Journal of Robust and Nonlinear Control, vol. 28, no. 4, pp. 1233-1245, 2018.

[39] J. Wu, Z.-G. Wu, J. Li, G. Wang, H. Zhao, and W. Chen, "Practical adaptive fuzzy control of nonlinear pure-feedback systems with quantized nonlinearity input," IEEE Transactions on Systems, Man, and Cybernetics: Systems, vol. 49, no. 3, pp. 638-648, 2019.

[40] M. Krstic, I. Kanellakopoulos, V. Petar, and Kokotovic, Nonlinear and Adaptive Control Design, Wiley, New York, NY, USA, 1995.

[41] Z. Wu, Y. Xia, and X. Xie, "Stochastic barbalat's lemma and its applications," IEEE Transactions on Automatic Control, vol. 57, no. 6, pp. 1537-1543, 2011.

[42] S. Xiao and J. Dong, "Robust adaptive fault-tolerant tracking control for uncertain linear systems with time-varying performance bounds," International Journal of Robust and Nonlinear Control, vol. 29, no. 46, pp. 849-866, 2019.

[43] Q. Hou, J. Dong, and C. Xi, "Adaptive neural network tracking control for switched uncertain non-linear systems with actuator failures and time-varying delays," IET Control Theory \& Applications, vol. 13, no. 12, pp. 1929-1939, 2019. 


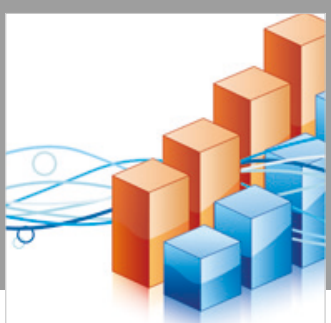

Advances in

Operations Research

\section{-n-m}
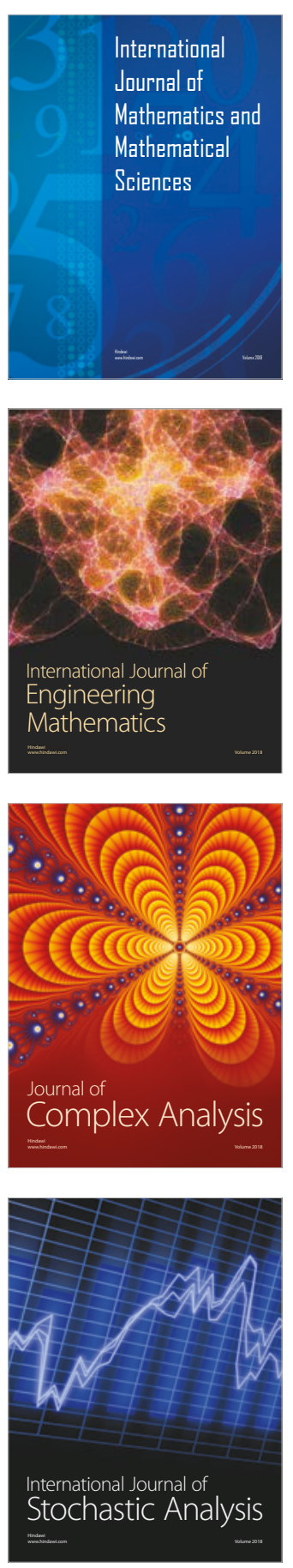
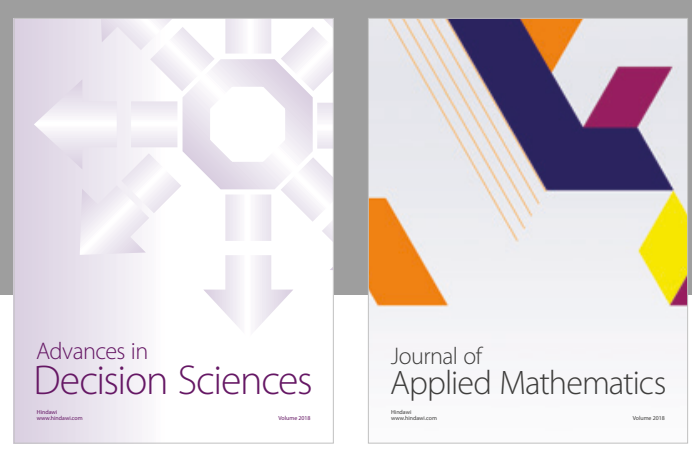

Journal of

Applied Mathematics
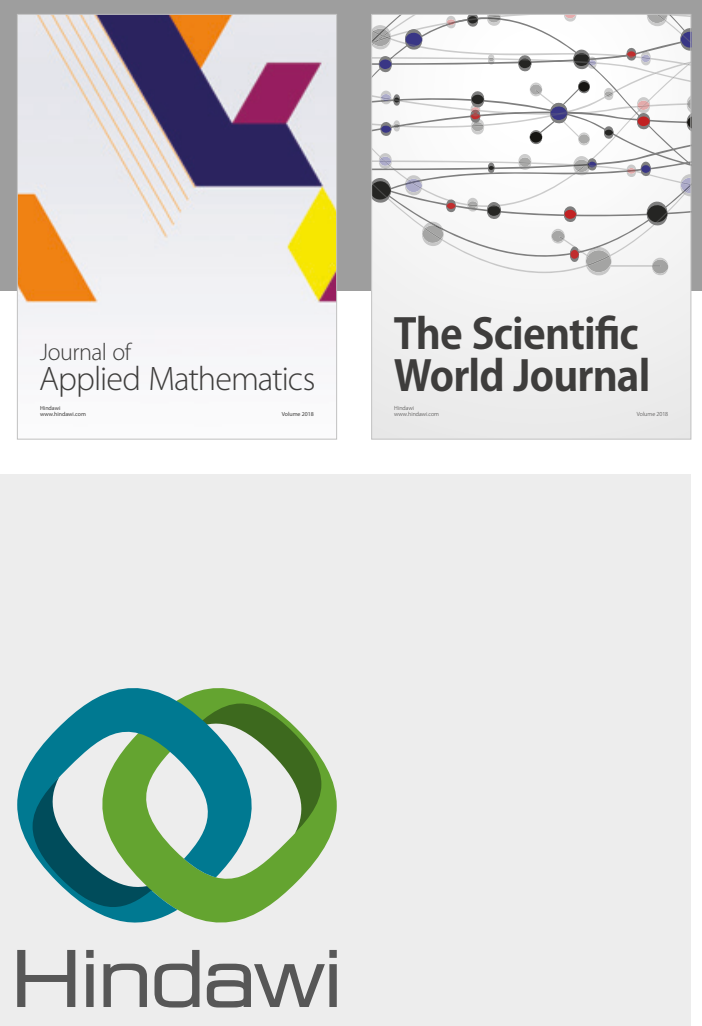

Submit your manuscripts at

www.hindawi.com

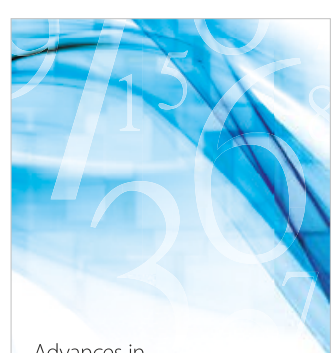

Advances in
Numerical Analysis
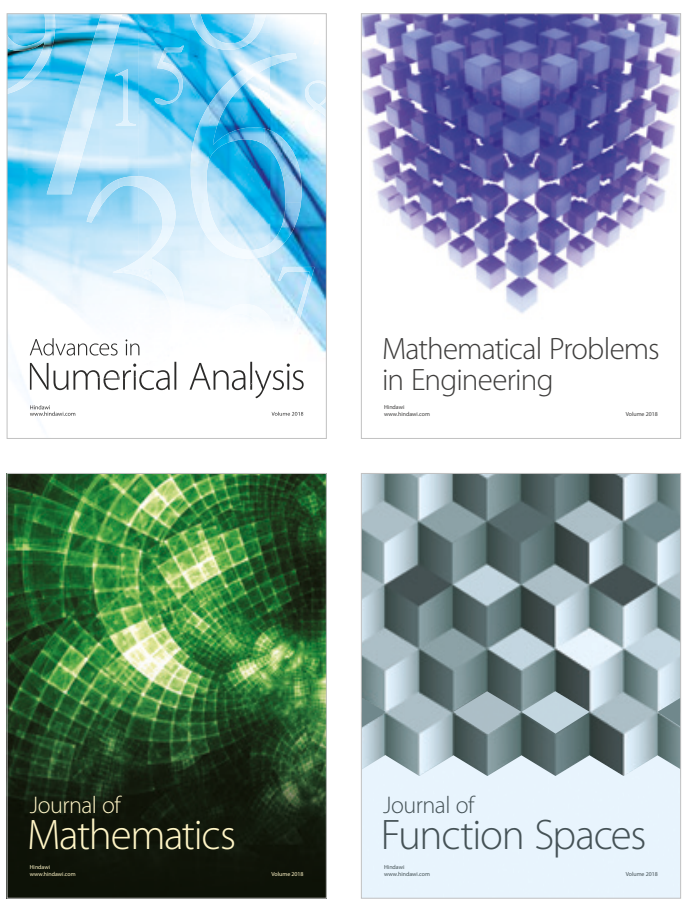

Mathematical Problems in Engineering

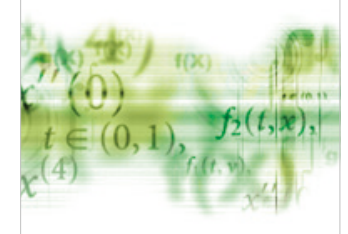

International Journal of

Differential Equations

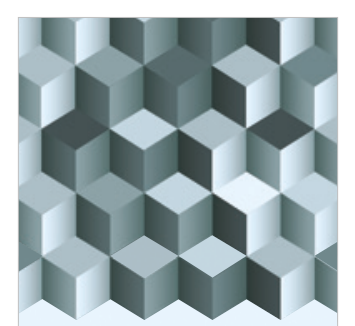

Journal of

Function Spaces
The Scientific

World Journal

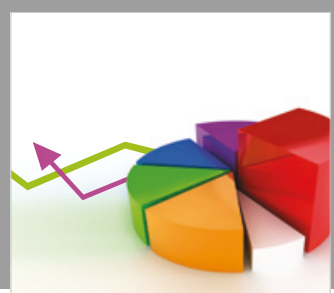

Journal of

Probability and Statistics
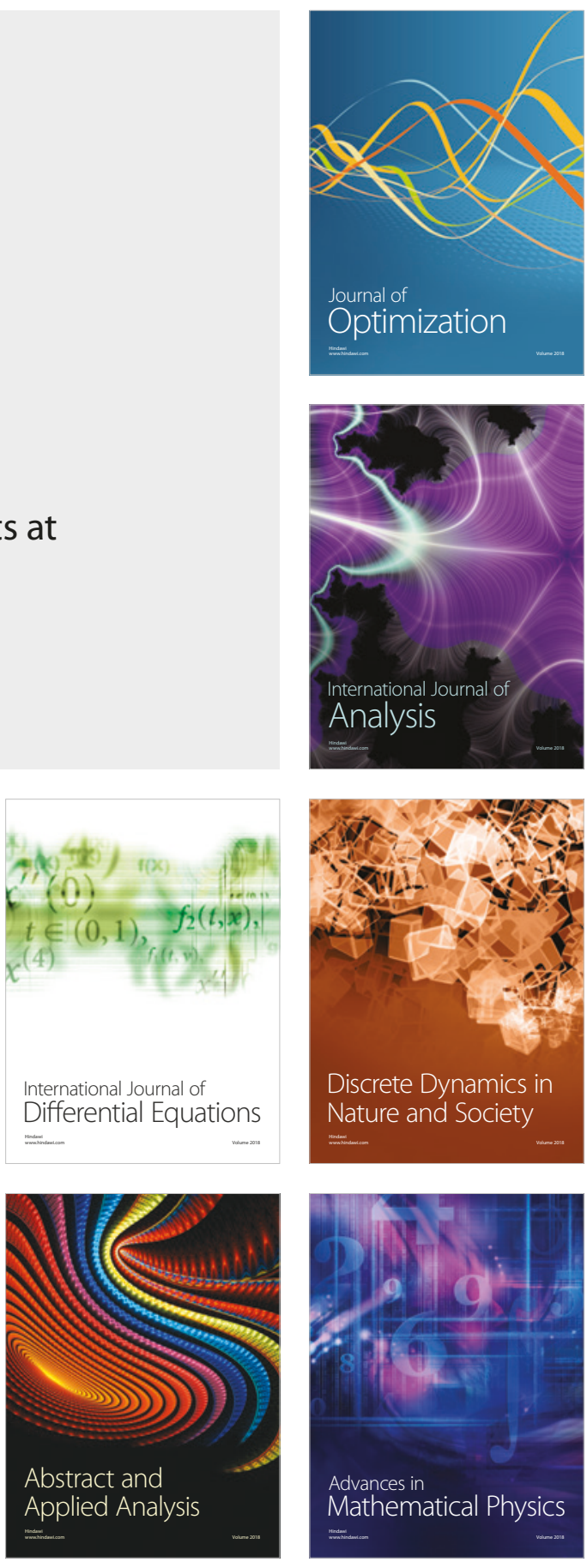